\title{
Spark is superior to Map Reduce over Big Data
}

\author{
Shaik Farook \\ M.Tech(CSE) \\ KHIT,Guntur \\ India
}

\author{
G. Lakshmi Narayana \\ Assistant Professor(Dept.of \\ CSE) \\ KHIT, Guntur, \\ India
}

\author{
B. Tarakeswara Rao, PhD \\ Professor(Dept.of CSE) \\ KHIT, Guntur \\ India
}

\begin{abstract}
In the Big Data group, MapReduce has been seen as one of the key empowering methodologies for taking care of ceaselessly expanding requests on figuring assets forced by Big Datasets yet at the same time numerous issues arrive with MapReduce keeping in mind the end goal to handle a much more extensive cluster of employments, combination into Hadoop's native file system. The purpose behind this is the high versatility of the MapReduce worldview which takes into account hugely parallel and circulated execution over an expansive number of figuring hubs. This paper address the how supplant MapReduce with Apache Spark as the default preparing for Hadoop.Apache Spark is superior to MapReduce towards leads issues and difficulties in taking care of Big Data with the target of giving an outline of the field, encouraging better arranging and administration of Enormous Information ventures, larger amount reflection and speculation of MapReduce.
\end{abstract}

\section{Keywords}

Big Data, Big Data Analytics, MapReduce, Apache Spark

\section{INTRODUCTION}

Late improvements in the Web, online networking, sensors and cell phones have brought about the blast of information set sizes. For instance, twitter today has more than one billion clients, with more than 600 million dynamic clients producing more than 600 terabytes of new information every day [1]. Customary information handling and stockpiling methodologies were outlined in a period when accessible equipment, stockpiling and preparing prerequisites were altogether different than they are today. Along these lines, those methodologies are confronting numerous difficulties in tending to Big data requests. The expression "Big data" alludes to expansive and complex information sets made up of an assortment of organized and unstructured information which are too enormous, too quick, or too difficult to be overseen by customary methods. Enormous Information is described by the 4Vs [2]: volume, speed, assortment, and veracity. Volume alludes to the amount of information, assortment alludes to the differences of information sorts, speed alludes both to how quick information are produced and how quick they must be prepared, and veracity is the capacity to believe the information to be precise and solid when settling on vital choices. Undertakings know that Big data can possibly effect center business procedures, give upper hand, and expand incomes [2]. In this way, associations are investigating approaches to improve utilization of Big data by examining them to discover important experiences which would prompt better business choices and enhance their business. MapReduce is an exceedingly adaptable programming worldview equipped for preparing huge volumes of information by method for parallel execution on an extensive number of product figuring hubs. It was as of late advanced by Google [3], however today the MapReduce worldview has been actualized in numerous open source extends, the most unmistakable being the Apache Hadoop [4].

The fame of MapReduce can be certify to its high versatility, adaptation to non-critical failure, effortlessness and autonomy from the programming dialect or the information stockpiling system. In the Enormous Information group, MapReduce has been seen as one of the key empowering methodologies for taking care of the consistently expanding requests on figuring assets forced by gigantic information sets. In the meantime, MapReduce confronts various deterrents when managing Big data including the absence of an abnormal state dialect, for example, SQL, challenges in executing iterative calculations, support for iterative specially appointed information investigation, and stream preparing. In order to address the issues with MapReduce we proposed novel substitution for MapReduce is Apache Spark. This paper intends to recognize how the Apache Spark is superior to anything MapReduce towards leads issues and difficulties in taking care of Enormous Information with the goal of giving a diagram of the field, encouraging better arranging and administration of Big data ventures, larger amount deliberation and speculation of MapReduce.

\section{MAPREDUCE OVERVIEW}

MapReduce is a programming worldview for preparing Big Data sets in circulated situations [3]. In the MapReduce worldview, the Guide capacity performs separating and sorting, while the Lessen capacity does gathering and conglomeration operations. The 'welcome to India' of MapReduce is the word numbering sample: it checks the presence of every word in an arrangement of archives. The Map function splits the document into words and for each word in a document it produces a (key, value) pair.

\section{function map(name, document)}

for each word in document

emit (word, 1)

The Decrease capacity is in charge of accumulating data got from Guide capacities. For every key, word, the Lessen capacity takes a shot at the rundown of qualities, partialCounts. To compute the event of every word, the Decrease capacity bunches by word and wholes the qualities got in the partialCounts list. Capacity lessen (word, List partialCounts) whole $=0$ for every $\mathrm{pc}$ in partialCounts total $+=$ pc radiate (word, total) the last yield is the rundown of words with the number of appearance of every word. Figure 1 outlines the MapReduce stream. One hub is chosen to be the expert in charge of doling out the work, while the rest are laborers. The information is partitioned into parts and the expert allots parts to Guide laborers. Every specialist forms the relating data split, creates key/quality matches and thinks of them to transitional files (on plate or in memory). The expert informs the Lessen specialists about the area of the middle files and the Decrease laborers read information, 
process it as indicated by the Diminish capacity, lastly, and compose information to output files.

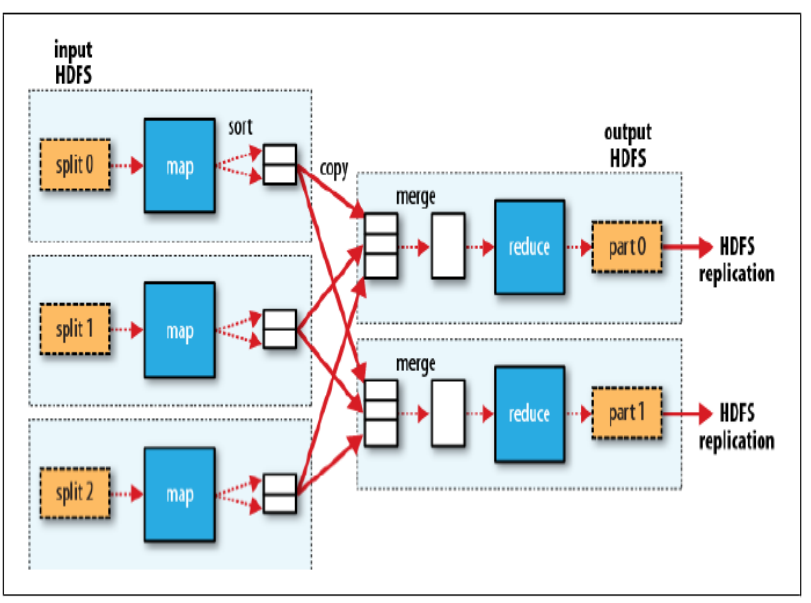

Figure 1. MapReduce flow

This diagram makes it clear why the information stream in the middle of guide and decrease assignments is informally known as "the mix," as each diminish errand is bolstered by numerous guide undertakings. The mix is more convoluted than this chart recommends, and tuning it can bigly affect work execution time.

The primary commitment of the MapReduce worldview is adaptability as it takes into account very parallelized and appropriated execution over an extensive number of hubs. In the MapReduce worldview, the Guide or Diminish errand is partitioned into a high number of occupations which are relegated to hubs in the system. Unwavering quality is accomplished by reassigning any fizzled hub's business to another hub. An understood open source MapReduce execution is Hadoop which actualizes MapReduce on top of Hadoop Distributed File System (HDFS).

Table I. An Overview Of Mapreduce Challenges

\begin{tabular}{|c|c|c|}
\hline & Main challenges & Main solution approaches \\
\hline \multirow{4}{*}{$\begin{array}{l}\text { Data } \\
\text { Storage }\end{array}$} & \multirow{2}{*}{$\begin{array}{l}\text { Schema-free, index- } \\
\text { free }\end{array}$} & In-database MapReduce \\
\hline & & $\begin{array}{l}\text { NoSQL stores - MapReduce with various } \\
\text { indexing approaches }\end{array}$ \\
\hline & \multirow{2}{*}{$\begin{array}{l}\text { Lack of standardized } \\
\text { SQL-like language }\end{array}$} & Apache Hive -SQL on top of Hadoop \\
\hline & & $\begin{array}{l}\text { NoSQL stores: proprietary SQL-like } \\
\text { languages (Cassandra, MongoDB) or Hive } \\
\text { (HBase) }\end{array}$ \\
\hline \multirow[t]{4}{*}{ Analytics } & $\begin{array}{l}\text { Scaling complex } \\
\text { linear algebra }\end{array}$ & $\begin{array}{l}\text { Use computationally less expensive, though } \\
\text { less accurate, algebra }\end{array}$ \\
\hline & Interactive analysis & $\begin{array}{l}\text { Map interactive query processing } \\
\text { techniques for handling small data, to } \\
\text { MapReduce }\end{array}$ \\
\hline & Iterative algorithms & $\begin{array}{l}\text { Extensions of MapReduce implementation } \\
\text { such as Twister and HaLoop }\end{array}$ \\
\hline & \begin{tabular}{|l|}
$\begin{array}{l}\text { Statistical challenges } \\
\text { for learning }\end{array}$ \\
\end{tabular} & Data pre-processing using MapReduce \\
\hline \multirow[t]{2}{*}{$\begin{array}{l}\text { Online } \\
\text { processing }\end{array}$} & \begin{tabular}{|l|l} 
Performance / \\
Latency issues
\end{tabular} & $\begin{array}{l}\text { Direct communication between phases and } \\
\text { jobs }\end{array}$ \\
\hline & Programming model & $\begin{array}{l}\text { Alternative models, such as MapUpdate } \\
\text { and Twitter's Storm }\end{array}$ \\
\hline \multirow{3}{*}{$\begin{array}{l}\text { Privacy } \\
\text { and } \\
\text { security }\end{array}$} & Auditing & $\begin{array}{l}\text { Trusted third party monitoring, security } \\
\text { analytics }\end{array}$ \\
\hline & Access control & $\begin{array}{l}\text { Optimized access control approach with } \\
\text { semantic understanding }\end{array}$ \\
\hline & Privacy & $\begin{array}{l}\text { Privacy policy enforcement with security to } \\
\text { prevent information leakage }\end{array}$ \\
\hline
\end{tabular}

\section{APACHE SPARK}

Spark is another execution system. Like MapReduce, it works with the filesystem to disperse your information over the group, and process that information in parallel. Like MapReduce, it likewise takes an arrangement of directions from an application composed by a designer. MapReduce was for the most part coded from Java; Sparkle backings Java, as well as Python and Scala, which is a more up to date dialect that contains some alluring properties for controlling information

\subsection{Major difference among MapReduce and Spark}

Spark tries to keep things in memory, whereas MapReduce keeps shuffling things in and out of disk

MapReduce embeds obstructions, and it sets aside quite a while to compose things to circle and read them back. Thus MapReduce can be moderate and difficult. The disposal of this confinement makes Sparkle requests of extent quicker. For things like SQL motors, for example, Hive, a chain of MapReduce operations is generally required, and this requires a ton of I/O action. On to plate, off of circle on to plate, off of circle. At the point when comparative operations are keeping running on Spark, Sparkle can keep things in memory without $\mathrm{I} / \mathrm{O}$, and so you can continue working on the same information rapidly

It's easier to develop for Spark. Spark is substantially more intense and expressive as far as how you give it directions to crunch information. Sparkle has a Guide and a Decrease capacity like MapReduce, however it includes others like Channel, Join and Gathering by, so it's simpler to produce for Spark. Truth be told, Sparkle accommodates loads of guidelines that are a more elevated amount of deliberation than what MapReduce presented

How Spark is compatible with Apache hadoop?

- Spark can keep running on Hadoop 2's YARN bunch supervisor,

- Spark can read any current Hadoop information.

- Hive, Pig and Mahout can keep running on Spark.

- Advanced Big Data Analyticsis perplexing.

- Hadoop MapReduce (MR) works really well in the event that you can express your issue as a solitary MR work. Practically speaking, most issues don't fit flawlessly into a solitary MR work.

- MR is moderate for cutting edge Enormous Information Examination, for example, iterative preparing and storing of datasets which are appropriate to machine learning. Sparkle enhances MapReduce by uprooting the need to compose information to plate between steps.

- $\quad$ Need to coordinate numerous different apparatuses for cutting edge Big Data Analyticsfor Questions, Gushing Examination, Machine Learning and Chart 


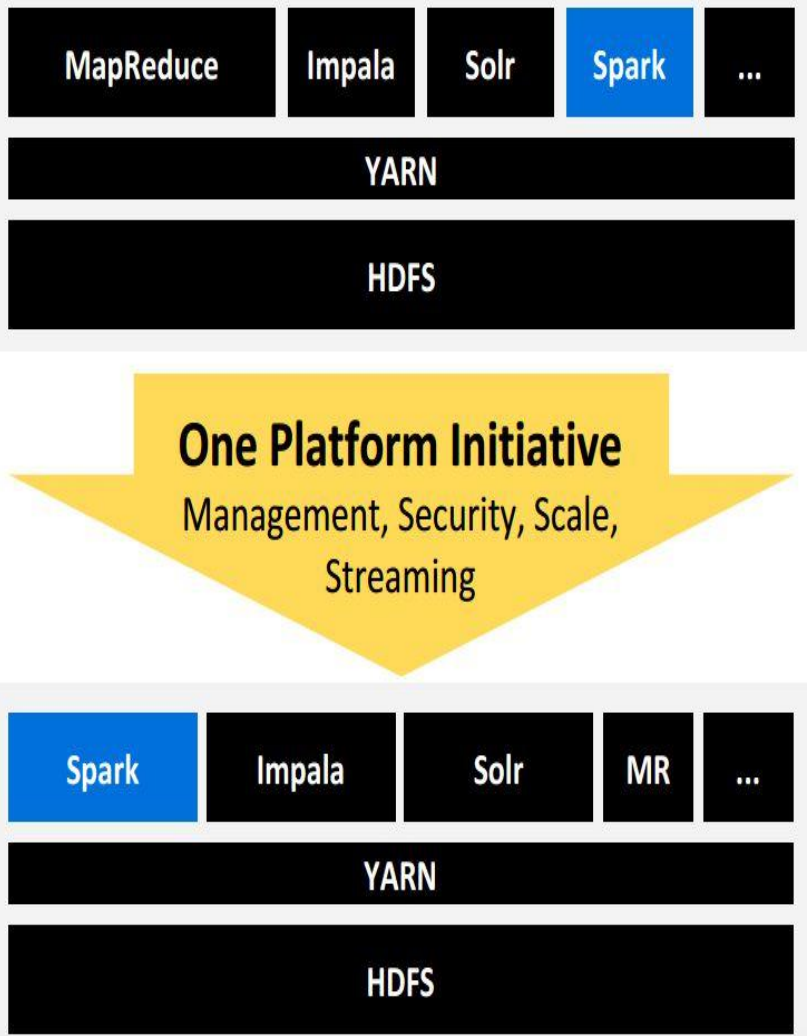

Figure 2. Apache Hadoop framework
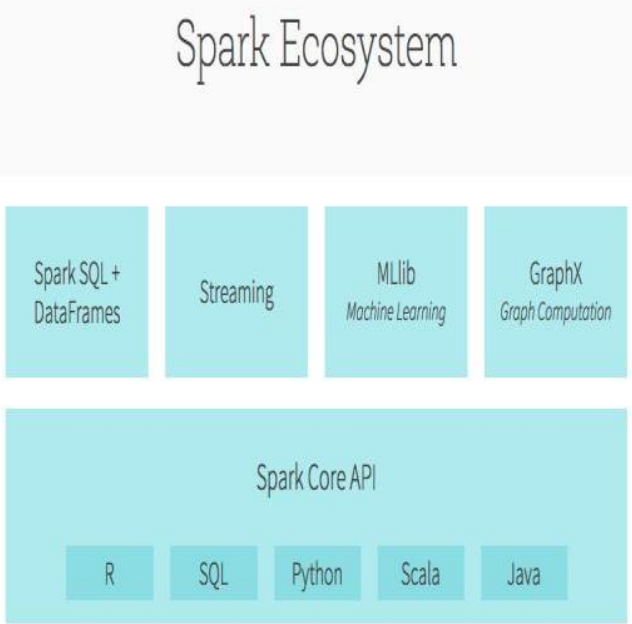

Figure 3. Data bricks

Huge amounts of individuals are doing information incorporation and ETL on MapReduce, and also cluster calculation, machine learning and clump investigation. Yet, these things will be much quicker on Spark. Intelligent examination and BI are conceivable on Sparkle, and the same goes for ongoing stream preparing. So a portion of the new utilizes cases are simply the old use cases, done quicker, while some are absolutely new. There are a few things that just couldn't have been finished with worthy execution on MapReduce.

\subsection{Key capabilities of Spark}

- Fast parallel data processing as intermediate data is stored in memory and only persisted to disc if needed.

- Apache Spark provides higher level abstraction and generalization of MapReduce. Over 80 high level built-in operators. Besides MapReduce, Spark supports SQL queries, streaming data, and complex analytics such asmachine learning and graph algorithms out-of-the-box.

- MapReduce is limited to batch processing. Spark lets you write streaming and batch-mode applications with verysimilar logic and APIs instead of using different tools.

- Apache Spark provides a set of composable building blocks for writing in Scala, Java or Python, concise queries and data flows. This makes developers highly productive.

- It is possible to build a single data workflow that leverages, streaming, batch, sql and machine learning for example.

- Spark runs on Hadoop, Mesos, standalone, or in the cloud. It can access diverse data sources including HDFS, Cassandra, HBase, S3.

\subsection{Features in Spark over MapReduce (Developing Perspective)}

\section{Language Flexibility}

At the point when creating in MapReduce, you are regularly compelled to join together essential operations as custom Mapper/Reducer employments in light of the fact that there are no inherent elements to rearrange this procedure. Therefore, numerous designers swing to the more elevated amount APIs offered by structures like Apache Crunch or Falling to compose their MapReduce jobs.In difference, Sparknatively gives a rich and constantly developing library of administrators

\section{APIs Matches with Goals}

At the point when creating in MapReduce, you are regularly compelled to join together essential operations as custom Mapper/Reducer employments in light of the fact that there are no inherent elements to rearrange this procedure. Therefore, numerous designers swing to the more elevated amount APIs offered by structures like Apache Crunch or Falling to compose their MapReduce jobs.In difference, Spark natively gives a rich and constantly developing library of artesian,cogroup,collect,count,countByValue,distinct,filter,flat Map,fold,groupByKey,join,map,mapPartitions,reduce,reduce ByKey,sample,sortByKey,subtract,take,union

\section{Automatic Parallelization of Complex Flows}

While developing an unpredictable pipeline of MapReduce occupations, the errand of effectively parallelizing the succession of employments is left to you. Along these lines, a scheduler instrument, for example, Apache Oozie is frequently required to painstakingly develop this grouping. With Spark, an entire arrangement of individual undertakings is communicated as a solitary project stream that is sluggishly assessed so that the system has a complete photo of the execution diagram. This methodology permits the center scheduler to effectively outline conditions crosswise over 
diverse platformss in the application, and consequently parallelize the stream of administrators without client intercession

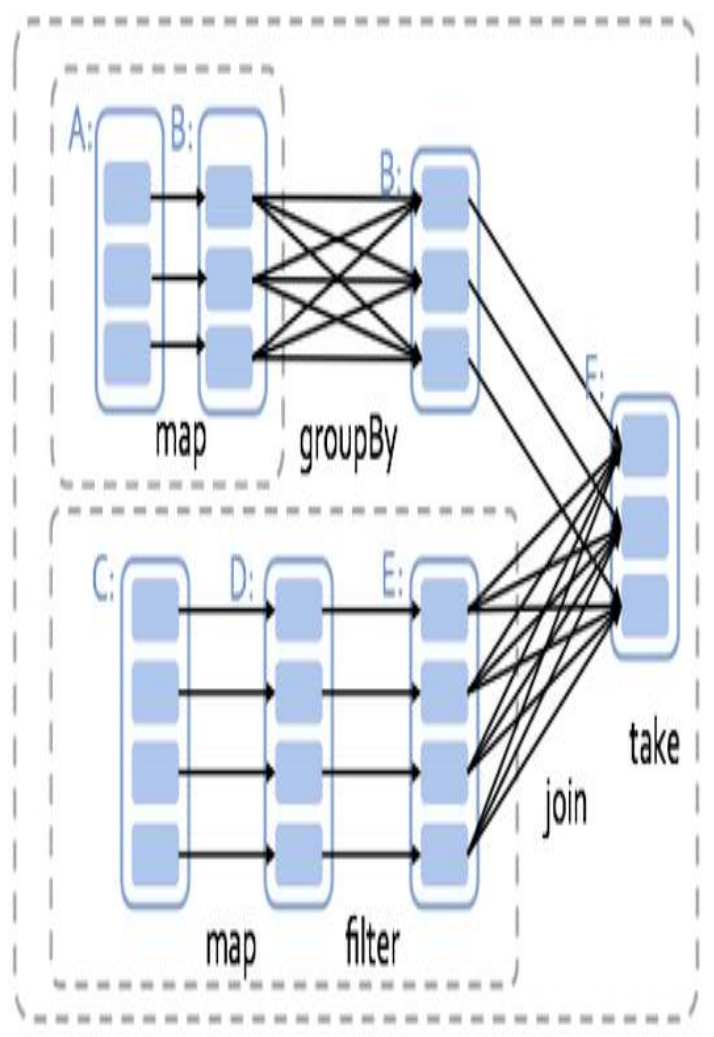

Figure 4.Complex flow of Simple application

This straightforward application communicates a perplexing stream of six stages. In any case, the genuine stream is totally escaped the client the system consequently decides the right parallelization crosswise over platforms and builds the chart accurately. Conversely, exchange motors would oblige you to physically develop the whole chart and also show the correct parallelization

\section{Interactive Shell}

Spark additionally gives you a chance to get to your datasets through a straightforward yet particular Spark shell for Scala and Python. With the Spark shell, engineers and clients can begin getting to their information and controlling datasets without the full exertion of composing a conclusion to-end application.

\section{PERFORMANCE}

While this post has concentrated on how Spark enhances execution as well as programmability, we shouldn't disregard one of the most ideal approaches to make designers more proficient: execution! Engineers regularly need to run applications many times over the improvement cycle, working with subsets of information and in addition full information sets to more than once take after the create/test/troubleshoot cycle. In a Big Data connection, each of these cycles can be exceptionally burdensome, with every test cycle, for instance, being hours long. While there are different routes systems to mitigate this issue, one of the best is to just run your project quick. On account of the execution advantages of Sparkle, the improvement lifecycle can be really abbreviated simply because of the way that the test/investigate cycles are much shorter.

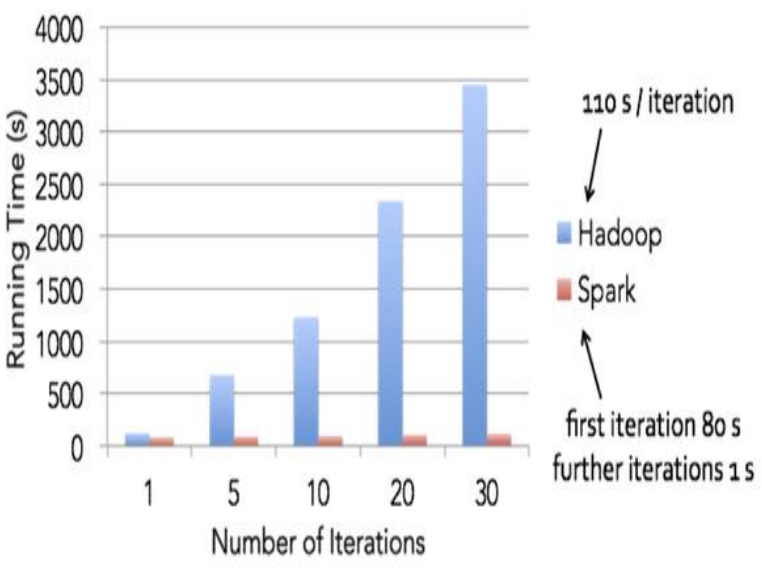

Figure 5.Performance of Spark

\section{CONCLUSIONS}

In this paper we reviewed the MapReduce impediments in meeting the consistently expanding registering requests of Big Data. This work concentrated on substitution of MapReduce strategy, one of the key empowering methodologies for taking care of Enormous Information requests by method for profoundly parallel preparing on countless hubs. Issues and difficulties MapReduce confronts when managing Enormous Information are leads with Apache Spark it has comparable levels of security, sensibility, and versatility as MapReduce, and ought to be similarly incorporated with whatever remains of the innovations that include the perpetually growing Hadoop platforms

\section{REFERENCES}

[1] P. Zadrozny and R. Kodali, Big Data Analytics using Splunk, Berkeley, CA, USA: Apress, 2013. [2] F. Ohlhorst, Big Data Analytics: Turning Big Data into Big Money, Hoboken, N.J, USA: Wiley, 2013.

[2] J. Dean and S. Ghemawat, "MapReduce: Simplified data processing on large clusters," Commun ACM, 51(1), pp. 107-113, 2008.

[3] Apache Hadoop, http://hadoop.apache.org.

[4] F. Li, B. C. Ooi, M. T. Özsu and S. Wu, "Distributed data management using MapReduce," ACM Computing Surveys, 46(3), pp. 1-42, 2014.

[5] C. Doulkeridis and K. Nørvåg, "A survey of large-scale analytical query processing in MapReduce," The VLDB Journal, pp. 1-26, 2013.

[6] P. Bhatotia, A. Wieder, R. Rodrigues, U. A. Acar and R. Pasquin, "Incoop: MapReduce for incremental computations," Proc. of the 2nd ACM Symposium on Cloud Computing, 2011.

[7] Y. Chen, S. Alspaugh and R. Katz, "Interactive analytical processing in Big Data systems: A cross-industry study of MapReduce workloads," Proc. of the VLDB Endowment, 5(12), pp. 1802-1813, 2012.

[8] http://www.sparkbigdata.com/102-spark-blog-slimbaltagi/1-getting-started

[9] S. Melnik, A. Gubarev, J. J. Long, G. Romer, S. Shivakumar, M. Tolton and T. Vassilakis, "Dremel: Interactive analysis of Web-scale datasets," Proc. of the VLDB Endowment, 3(1-2), pp. 330-339, 2010. 